Pacific Journal of Mathematics

MONOTONE MAPPINGS OF A TWO-DISK ONTO ITSELF
WHICH FIX THE DISKS BOUNDARY CAN BE CANONICALLY
APPROXIMATED BY HOMEOMORPHISMS WILLIAM EMERY HAVE 


\title{
MONOTONE MAPPINGS OF A TWO-DISK ONTO ITSELF WHICH FIX THE DISK'S BOUNDARY CAN BE CANONICALLY APPROXIMATED BY HOMEOMORPHISMS
}

\author{
WILLIAM E. HAVER
}

\begin{abstract}
The theorem stated in the title is proven. As a corollary it is shown that the space of all such monotone mappings is an absolute retract.
\end{abstract}

1. Introduction. Let $D^{n}$ denote the standard $n$-ball of radius one in $E^{n}$ and $H\left(D^{n}\right)$ the space of all homeomorphisms of $D^{n}$ onto itself which equal the identity on the boundary of $D^{n}$. Let $\overline{H\left(D^{n}\right)}$ denote the space of all mappings of $D^{n}$ onto itself which can be approximated arbitrarily closely by elements of $H\left(D^{n}\right)$. Under the supremum topology, $H\left(D^{n}\right)$ and $\overline{H\left(D^{n}\right)}$ are separable metric spaces; $\overline{H\left(D^{n}\right)}$ is complete under the supremum metric. It is known that $\overline{H\left(D^{n}\right)}$ is locally contractible [7], $\overline{H\left(D^{n}\right)} \times l_{2} \approx \overline{H\left(D^{n}\right)}$ [4], $\overline{H\left(D^{n}\right)}$ is homogeneous [7], and that $\overline{H\left(D^{1}\right)} \approx l_{2}$ [3]. In this paper we shall be concerned with the case $n=2$, and to simplify notation we shall write $D$ for $D^{2}$. It is wellknown (cf. [8]) that $\overline{H(D)}$ is the space of all monotone mappings of $D$ onto itself which equal the identity when restricted to the boundary of $D$.

We shall show [Theorem 1] that the elements of $\overline{H(D)}$ can be "canonically approximated" by elements of $H(D)$ and [Theorem 2] that $\overline{H(D)}$ is an absolute retract. The work of this paper depends heavily on W. K. Mason's paper, "The space of all self-homeomorphisms of a two-cell which fix the cell's boundary is an absolute retract", [9]. The crux of Mason's paper is the definition of a basis for $H(D)$ which can be shown to possess some particularly nice properties. We shall review the definition of this basis in the following section and then define a basis for $\overline{H(D)}$. Familiarity will be assumed with the notation and basic definitions of [9].

2. Mason's basis for $H(D)$. Consider $D$ to be a rectangle in $R^{2}$ with horizontal and vertical sides. A grating, $P$, on $D$ consists of a finite number of spanning segments (crosscuts) across $D$, parallel to its sides, with the same number of horizontal and vertical crosscuts. Let $P_{1}, P_{2}, \cdots$ be a sequence of gratings on $D$ such that (a) the mesh of $P_{i}$ approaches 0 as $i$ increases and (b) if $l$ is a crosscut of $P_{i}$ and $j \geqq i$, then $l$ is a crosscut of $P_{j}$.

Let $\mathscr{H}$ be the collection of all polyhedral disks $H$ contained in $D$ 
such that $\mathrm{Bd}(H)$ is the union of a vertical segment in the left side of $\mathrm{Bd}(D)$, a vertical segment in the right side of $\mathrm{Bd}(D)$, a polygonal spanning arc of $D, H^{T}$, that is contained in the closure of the same component of $H(D)-H$ as the top of $\mathrm{Bd}(D)$, and a polygonal spanning arc of $D, H^{B}$, that is contained in the closure of the same component of $H(D)-H$ as the bottom of $\mathrm{Bd}(D)$. Let $\mathscr{Y}$ be the collection of all polyhedral disks $V$ contained in $D$ such that $\mathrm{Bd}(V)$ is the union of a horizontal segment in the top of $\mathrm{Bd}(D)$, a horizontal segment in the bottom of $\mathrm{Bd}(D)$, a polygonal spanning arc of $D, V^{L}$, that is contained in the closure of the same component of $H(D)-V$ as the left side of $\mathrm{Bd}(D)$ and a polygonal spanning arc of $D, V^{R}$, that is contained in the closure of the same component of $H(D)-V$ as the right side of $\mathrm{Bd}(D)$.

Let $P_{j}$ be a grating from the sequence $P_{1}, P_{2}, \cdots$. Let $\left\{l_{1}, \cdots\right.$, $\left.l_{n}\right\}$ be the set of horizontal crosscuts of $P_{j}$ and $\left\{m_{1}, \cdots, m_{n}\right\}$ the set of vertical crosscuts. Let $\left\{H_{1}, \cdots, H_{n}\right\} \subset \mathscr{H}$ satisfy $H_{i} \cap H_{j}=\varnothing$ if $i \neq j$ and $\left\{V_{1}, \cdots, V_{n}\right\} \subset \mathscr{V}$ satisfy $V_{i} \cap V_{j}=\varnothing$ if $i \neq j$. Then define

$$
\begin{aligned}
& O\left(P_{j} ; H_{1}, \cdots, H_{n} ; V_{1}, \cdots, V_{n}\right) \\
&=\left\{f \in H(D) \mid f\left(l_{i}\right) \subset H_{i}-\left\{\mathrm{Cl}\left(D-H_{i}\right)\right\}\right. \text { and } \\
&\left.f\left(m_{i}\right) \subset V_{i}-\left\{\mathrm{Cl}\left(D-V_{i}\right)\right\} \text { for } 1 \leqq i \leqq n\right\} .
\end{aligned}
$$

Then the basis for $H(D)$, which Mason denotes $H V T$, is the collection of all such open sets.

3. A Basis for $\overline{H(D)}$. In this section we define a basis, $\beta$, for $\overline{H(D)}$ and demonstrate that it possesses some nice properties. Let $P_{j}$, $\left\{H_{1}, \cdots, H_{n}\right\}$ and $\left\{V_{1}, \cdots, V_{n}\right\}$ be as in the definition of $H V T$. The basis, $\beta$, will consist of all sets of the following form:

$$
\begin{aligned}
B( & \left.P_{j} ; H_{1}, \cdots, H_{n} ; V_{1}, \cdots, V_{n}\right) \\
\quad= & \left\{f \in \overline{H(D)} \mid f^{-1}\left(l_{i}\right) \subset H_{i}-\left\{\mathrm{Cl}\left(D-H_{i}\right)\right\}\right. \text { and } \\
& \left.f^{-1}\left(m_{i}\right) \subset V_{i}-\left\{\mathrm{Cl}\left(D-V_{i}\right)\right\}, \text { for } 1 \leqq i \leqq n\right\} .
\end{aligned}
$$

We note that $f \in B\left(P_{j} ; H_{1}, \cdots, H_{n} ; V_{1}, \cdots, V_{n}\right) \cap H(D)$ if and only if $f^{-1} \in O\left(P_{j} ; H_{1}, \cdots, H_{n} ; V_{1}, \cdots, V_{n}\right)$. To see that the elements of $\beta$ are open subsets of $\overline{H(D)}$, let $f$ be an arbitrary element of $B\left(P_{j} ; H_{1}\right.$, $\left.\cdots, H_{n} ; V_{1}, \cdots, V_{n}\right)$. Then let

$$
\varepsilon=\min _{1 \leqq i \leqq n}\left\{d\left(f\left(H_{i}^{T} \cup H_{i}^{B}\right), \bigcup_{j=1}^{n} l_{j}\right), d\left(f\left(V_{i}^{L} \cup V_{i}^{R}\right), \bigcup_{j=1}^{n} m_{j}\right)\right\} .
$$

Let $g$ be an arbitrary element of $\overline{H(D)}$ satisfying $d(f, g)<\varepsilon$. Suppose that $g \notin B\left(P_{j} ; H_{1}, \cdots, H_{n} ; V_{1}, \cdots, V_{n}\right)$. Then, without loss of generality, we can assume that there exists an integer $i$ such that $g^{-1}\left(l_{i}\right)$ 
is not contained in $H_{i}-\mathrm{Cl}\left(D-H_{i}\right)$. Since (i) $g^{-1}\left(l_{i}\right) \cap(\mathrm{Bd} D)=$ $f^{-1}\left(l_{i}\right) \cap(\mathrm{Bd} D) \subset H_{i}-\mathrm{Cl}\left(D-H_{i}\right)$, (ii) $g^{-1}\left(l_{i}\right)$ is a connected set and (iii) $H_{i}^{T} \cup H_{i}^{B}$ separates $H_{i}-\mathrm{Cl}\left(D-H_{i}\right)$ from $D-H_{i}$, there is an $x \in H_{i}^{T} \cup H_{i}^{B}$ such that $g(x) \in l_{i}$. But this implies that $d(f(x), g(x)) \geqq$ $d\left(f\left(H_{i}^{T} \cup H_{i}^{B}\right), l_{i}\right) \geqq \varepsilon$ and hence $d(f, g) \geqq \varepsilon$.

\section{Lemma 1. $\beta$ is a basis for $\overline{H(D)}$.}

Proof. Let $f \in \overline{H(D)}$ and $\varepsilon>0$ be given. We wish to find $B \in \beta$ such that $f \in B$ and $d(f, g)<\varepsilon$ for all $x \in B$. Pick a grating, $P_{j}$, such that diam $\left|\operatorname{st}\left(x, P_{j}\right)\right|<\varepsilon$ for every $x \in D$. Let $l_{i}$ be the $i$ th crosscut from the top of $D$. Choose $H_{1}^{T}$ to be a polygonal spanning arc of $D$ with one endpoint in each side of $D$ that separates the top of $D$ from $f^{-1}\left(l_{1}\right)$. Choose $H_{1}^{B}$ to be a polygonal spanning arc of $D$ that separates $f^{-1}\left(l_{1}\right)$ from $f^{-1}\left(l_{2}\right)$. The polyhedral disk $H_{1}$ is thus uniquely defined. Assume inductively that disks $H_{1}, \cdots, H_{i-1}$ have been defined in such a manner that $H_{j} \cap H_{k}=\varnothing$ if $1 \leqq j \leqq i-1,1 \leqq k \leqq i-1$, and $j \neq k$ and that for each $j, 1 \leqq j \leqq i-1, H_{j}^{T}$ separates $H_{j-1}^{B}$ from $f^{-1}\left(l_{j}\right)$ and $H_{j}^{B}$ separates $f^{-1}\left(l_{j}\right)$ from $f^{-1}\left(l_{j+1}\right)$. Choose $H_{i}^{T}$ to be a polygonal spanning arc of $D$ that separates $H_{i-1}^{B}$ from $f^{-1}\left(l_{i}\right)$. Finally choose $H_{i}^{B}$ to be a pylygonal spanning arc of $D$ that separates $f^{-1}\left(l_{i}\right)$ from $f^{-1}\left(l_{i+1}\right)$ (or from the bottom of $D$ if $i=n$ ). We have thus uniquely defined $H_{i}$ in such a way that the inductive hypothesis is satisfied. Define $V_{1}, \cdots, V_{n}$ in a similar manner. Now, by construction $f \in B\left(P_{j} ; H_{1}, \cdots, H_{n} ; V_{1}, \cdots, V_{n}\right)$ and if $g \in B\left(P_{j} ; H_{1}, \cdots, H_{n} ; V_{1}, \cdots\right.$, $\left.V_{n}\right)$ then $d(f, g)<\varepsilon$.

Lemma 2. Let $B_{1}, \cdots, B_{j}$ be elements of $\beta$. Then $B=\bigcap_{k=1}^{j} B_{k}$ is an element of $\beta$.

Proof. Assume $B \neq \varnothing$ and that $P_{k}$ is the grating associated with $B_{k}, 1 \leqq k \leqq j$. Hence for any $k, 1 \leqq k \leqq j$, every crosscut of $P_{k}$ is a crosscut of $P_{j}$. Let $l_{1}$ be the first horizontal crosscut of $P_{j}$. For each $k, 1 \leqq k \leqq j$, let $H_{1, k}$ be the element of $\mathscr{H}$ associated with $l_{1}$ and $B_{k}$ (if there is one). Let $H_{1}$ be the component of $D-\left(\bigcup_{k=1}^{j} H_{1, k}^{T} \cup \bigcup_{k=1}^{j} H_{1, k}^{B}\right)$ that contains $f^{-1}\left(l_{1}\right)$. Define in an analogous manner $H_{2}, \cdots, H_{n}$ and $V_{1}, \cdots, V_{n}$. It is clear that $B\left(P_{j} ; H_{1}, \cdots, H_{n} ; V_{1}, \cdots, V_{n}\right)=\bigcap_{k=1}^{j} B_{k}$. The elements of $\left\{H_{1}, \cdots, H_{n}\right\}$ are pairwise disjoint since each $H_{i}$ is contained in $H_{i, n}$ and the elements of $\left\{H_{1, j}, \cdots, H_{n, j}\right\}$ are pairwise disjoint.

Lemma 3 will follow as a corollary to the following theorem of Mason. (The proof of this theorem constitutes the bulk of [9].)

Theorem (Mason). Let $U$ be an element of $H V T$ and $K$ a finite 
dimensional compact subset of $U$. Then there is an embedding $\psi$ of the cone over $K$ into $U$ such that $\psi(f, 0)=f$, for all $f \in K$.

Lemma 3. Let $B=B\left(P_{j} ; H_{1}, \cdots, H_{n} ; V_{1}, \cdots, V_{n}\right)$ be an element of $\beta$ and $K$ a finite dimensional compact subset of $B \cap H(D)$. Then there is an embedding $\lambda$ of the cone over $K$ into $B \cap H(D)$ such that $\psi(f, 0)=f$, for all $f \in K$.

Proof. Since $H(D)$ is a topological group, the function $G: H(D) \rightarrow$ $H(D)$ defined by $G(f)=f^{-1}$ is a homeomorphism. Therefore, by the note following the definition of $\beta, G(K)$ is a finite dimensional compact subset of $U\left(P_{j} ; H_{1}, \cdots, H_{n} ; V_{1}, \cdots, V_{n}\right)$. Hence by Mason's theorem there is an embedding $\psi$ of the cone over $G(K)$ into $U\left(P_{j} ; H_{1}, \cdots\right.$, $\left.H_{n} ; V_{1}, \cdots, V_{n}\right)$ such that $\psi(f, 0)=f$, for all $f \in G(K)$. Define $\lambda: K \times$ $I \rightarrow B \cap H(D)$ by $\lambda(k, t)=G^{-1}(\psi(G(k), t))$.

4. The main results. The following theorem shows that the elements of $\overline{H(D)}$ can be canonically approximated by elements of $H(D)$.

THEOREM 1. Let $\alpha$ be an open cover of $\overline{H(D)}$. Then there exists a locally finite polyhedron, $\mathscr{P}$, and maps $b: \overline{H(D)} \rightarrow \mathscr{P}, \psi: \mathscr{P} \rightarrow H(D)$, and $\theta: \overline{H(D)} \times I \rightarrow \overline{H(D)}$ such that

(a) for each $f \in \overline{H(D)}$, there is an element, $U_{f}$, of $\alpha$ such that $\theta(f, t) \in U_{f}$, for each $t \in I$,

(b) $\theta(f, 1)=f$, for each $f \in \overline{H(D)}$,

(c) $\theta(f, 0)=\psi b(f)$, for each $f \in \overline{H(D)}$,

(d) $\theta(f, t) \in H(D)$ for each $f \in \overline{H(D)}$ and $t \in[0,1)$.

Proof. Let $\alpha^{\prime}$ be an open barycentric refinement $\alpha$ (i.e., if $f \in \overline{H(D) \text {, }}$ $\left|s t\left(f, \alpha^{\prime}\right)\right|$ is contained in some element of $\left.\alpha\right)$. For each positive integer, $k$, let $\alpha_{k}$ be an open cover of $\overline{H(D)}$ such that

(i) $\alpha_{k}$ is a refinement of $\alpha^{\prime}$,

(ii) if $V \in \alpha_{k}$, diam $V<1 / k$,

(iii) if $V \in \alpha_{k}$, then $V \in \beta$.

We next define an open cover, $\eta$, of $\overline{H(D)} \times[0,1)$.

Let $\eta=\left\{V \times[0,1 / 2) \mid V \in \alpha_{1}\right\} \cup \bigcup_{k=2}^{\infty}\left\{V \times\left(\frac{2^{k}-3}{2^{k}}, \frac{2^{k}-1}{2^{k}}\right) \mid V \in \alpha_{k}\right\}$.

Let $\gamma$ be a countable refinement of $\eta$ such that

(a) if $h \in \gamma, s t(h, r)$ is a finite set,

(b) if $h \in \gamma$, then there is an element of $\eta, V \times J$, such that $|s t(h, \gamma)| \subset V \times J$.

Let $\mathscr{P}$ be the nerve of $\gamma$ and $B: \overline{H(D)} \times[0,1) \rightarrow \mathscr{P}$ be the standard 
barycentric map. Order the element of $\gamma$, and for each $h_{i} \in \gamma$, let $V_{i} \times J_{i}$ be an element of $\eta$ such that $\left|s t\left(h_{i}, \gamma\right)\right| \subset V_{i} \times J_{i}$. Note that $V_{i} \in \beta$.

We shall define a map $\psi: \mathscr{P} \rightarrow H(D)$ by induction on the skeletons of $\mathscr{P}$. For each vertex $\left(h_{i}\right)$ of $\mathscr{P}$, let $\psi^{0}\left(\left(h_{i}\right)\right)$ be an arbitrary element of $H(D)$ intersected with the projection of $h_{i}$ onto $\overline{H(D)}$.

Now assume that for $m=1,2, \cdots, n$ we have defined $\psi^{m}: \mathscr{P}^{m} \rightarrow$ $H(D)$ such that $\psi^{m}$ extends $\psi^{m-1}$ and for each simplex $\sigma^{m}=\left(h_{\lambda_{0}}, \cdots, h_{\lambda_{m}}\right)$ :

(a) $\psi^{m}\left(\sigma^{m}\right)$ is finite dimensional,

(b) $\psi^{m}\left(\sigma^{m}\right) \subset H(D) \cap\left\{V_{i} \mid h_{i} \subset \bigcap_{j=0}^{m} s t\left(h_{\lambda_{j}}, \gamma\right)\right\}$.

Now let $\sigma^{n+1}=\left\langle h_{0}, \cdots, h_{n+1}\right\rangle$ be any simplex of $\mathscr{P}^{n+1}$. Let

$$
U=\bigcap\left\{V_{i} \mid h_{i} \subset \bigcap_{j=0}^{n+1} s t\left(h_{j}, \gamma\right)\right\}
$$

Since each $V_{i}$ is an element of $\beta$, by Lemma $2, U \in \beta$. By the inductive hypothesis the image under $\psi^{n}$ of the boundary of $\sigma^{n+1}$ is a finite dimensional compact subset of $U \cap H(D)$, denoted $K$. By Lemma 3 there is an embedding

$$
\lambda: \subset(K) \rightarrow U \cap H(D)
$$

such that $\lambda(f, 0)=f$ for all $f \in K$. We consider $\sigma^{n+1}$ to be the cone over its boundary, and so for $(x, t) \in \sigma^{n+1}$, let $\psi^{n+1}(x, t)=\lambda\left(\psi^{n}(x), t\right)$.

Extending over each $n+1$ simplex in this manner gives $\psi^{n+1}: \mathscr{P}^{n+1} \rightarrow H(D)$ and completes the induction. Hence $\lim _{n \rightarrow \infty} \psi^{n}=$ $\psi: \mathscr{P} \rightarrow H(D)$ is continuous by the continuity of each $\psi^{n}$ and the local finiteness of $\mathscr{P}$.

Let $b: \overline{H(D)} \rightarrow \mathscr{P}$ be defined by $b(f)=B((f, 0))$.

We next define the homotopy $\theta: \overline{H(D)} \times I \rightarrow \overline{H(D)}$ in the following manner:

$$
\theta(f, t)=\left\{\begin{array}{l}
\psi(B(f, t)), \quad t \neq 1 \\
f, t=1
\end{array}\right.
$$

Conditions (b), (c), and (d) are obviously satisfied. We show simultaneously that $\theta$ is continuous and that for each $f \in \overline{H(D)}$ there is an element $U_{f}$ of $\alpha$ such that for each $t \in I, \theta(f, t) \in U_{f}$.

Suppose that $(f, t) \in \overline{H(D)} \times[0,1)$ and that $\left(2^{k}-3\right) / 2^{k}<t<\left(2^{k}-1\right) / 2^{k}$. Let $h_{0}$ be any element of $\gamma$ which contains $(f, t)$. By the definition of $\psi, \psi B(f, t) \in V_{0}$. But $V_{0} \in \alpha_{k-1} \cup \alpha_{k} \cup \alpha_{k+1}$ and therefore the diameter of $V_{0}$ is less than $1 /(k-1)$ which implies that $d(\psi B(f, t), f)<$ $1 /(k-1)$ and thereby that $\theta$ is continuous. Since each $\alpha_{k}$ is a refinement of $\alpha^{\prime}$, there exists an element of $\alpha^{\prime}, U_{(f, t)}$, such that 
$\{f\} \cup\{\psi B(f, t)\} \subset V_{0} \subset U_{(f, t)}$. Since $\alpha^{\prime}$ is a barycentric refinement of $\alpha$, there is some element, $U_{f}$, of $\alpha$ such that $\bigcup_{t \in[0,1)} U_{(f, t)} \subset U_{f}$ and hence $\theta(f, t) \in U_{f}$, for each $t \in I$.

The following result is an immediate corollary of Theorem 1 and a theorem of Hanner [5] which states that a metric space $X$ is an $A N R$ if given an arbitrary cover, $\alpha$, of $X$ there exists a locally finite polyhedron $\mathscr{P}$, maps $b: X \rightarrow \mathscr{P}, \psi: \mathscr{P} \rightarrow X$, and $\theta: X \times I \rightarrow X$ such that $\theta(x, 0)=\psi b(x)$ for all $x \in X, \theta(x, 1)=x$ for all $x \in X$ and for each $x \in X$ there is an element $U$ of $\alpha$ such that $\theta(x, t) \in U$ for all $t \in[0,1]$.

\section{THEOREM 2. $\overline{H(D)}$ is an absolute retract.}

Proof. By the preceding comments, $\overline{H(D)}$ is an $A N R$. But $\overline{H(D)}$ is contractible by the Alexander isotopy [1] applied to $\overline{H(D)}$. The theorem follows since every contractible absolute neighborhood retract is an absolute retract.

5. Applications. (a) The author has shown [6] that $\overline{H(M)}$, the space of all mappings of a compact manifold onto itself which can be approximated arbitrarily closely by homeomorphisms, is weakly locally contractible. Theorem 1 can be used [7] to show that for any compact 2-manifold, $M^{2}, \overline{H\left(M^{2}\right)}$ is locally contractible.

(b) A problem of current interest is whether $H(D)$ is homeomorphic to $l_{2}$; it can easily by shown using a result of Anderson [2] that if $\overline{H(D)}$ is homeomorphic to $l_{2}$, then $H(D)$ is homeomorphic to $l_{2}$. Perhaps the results of this paper and the fact that $\overline{H(D)}$ is complete under the usual metric will be helpful in showing that $\overline{H(D)}$ is homeomorphic to $l_{2}$.

(c) L. C. Siebenmann [10] has asked whether the inclusion map $i: H(M) \rightarrow \overline{H(M)}$ is a homotopy equivalence. Theorem 1 provides an affirmative answer to the question for the special case $i: H(D) \rightarrow \overline{H(D)}$.

Added in proof. Recent work of Torunczyk ("Absolute retracts as factors of normed linear spaces," Fund. Math., to appear) implies that since $\overline{H(D)}$ is an $A R$ and $H(D) \times l_{2} \approx \overline{H(D)}, \overline{H(D)}$ is in fact homeomorphic to $l_{2}$.

\section{REFERENCES}

1. J. W. Alexander, On the deformation of an $n$-cell, Proc. Nat. Acad. Sci., U.S.A., 9 (1923), 406-407.

2. R. D. Anderson, Strongly negligible sets in Frechet manifolds, Bull. Amer. Math. Soc., 75 (1969), 64-67.

3. R. Geoghegan, On spaces of homeomorphisms, embeddings, and functions I, Topology, 11 (1972), 159-177. 
4. R. Geoghegan and D. W. Henderson, Stable function spaces, to appear.

5. O. Hanner, Some Theorems on Absolute Neighborhood Retracts, Arkiv Fur Matematik, 1 (1951), 389-408.

6. W. Haver, Homeomorphisms and UV maps, Proc. of SUNY Bingh. Conf. on Monotone Mappings and Open Mappings, (1970), 112-121.

7. - The Closure of the Space of Homeomorphisms on a Manifold, Trans. Amer. Math. Soc., to appear.

8. J. Hocking, Approximations to monotone mappings on noncompact two-dimensional manifolds, Duke Math. J., 21 (1954), 639-651.

9. W. K. Mason, The space of all self-homeomorphisms of a 2-cell which fix the cell's boundary is an absolute retract, Trans. Amer. Math. Soc., 161 (1971), 185-206.

10. L. C. Siebenmann, Approximating cellular maps by homeomorphisms, Topology, 11 (1972), 271-294.

Received September 6, 1972. Research partially supported by NSF Grant GP. 33872.

UNIVERSITY OF TENNESSEE 



\section{PACIFIC JOURNAL OF MATHEMATICS}

\section{EDITORS}

RICHARD ARENS (Managing Editor)

University of California

Los Angeles, California 90024

\section{R. A. Beaumont \\ University of Washington \\ Seattle, Washington 98105}

\section{J. DugundjI*}

Department of Mathematics University of Southern California Los Angeles, California 90007

D. Gilbarg and J. Milgram

Stanford University

Stanford, California 94305

\section{ASSOCIATE EDITORS}
E. F. BeCKenBaCH
B. H. NeumanN
F. WOLF
K. YoSHIDA

\section{SUPPORTING INSTITUTIONS}

\author{
UNIVERSITY OF BRITISH COLUMBIA \\ CALIFORNIA INSTITUTE OF TECHNOLOGY \\ UNIVERSITY OF CALIFORNIA \\ MONTANA STATE UNIVERSITY \\ UNIVERSITY OF NEVADA \\ NEW MEXICO STATE UNIVERSITY \\ OREGON STATE UNIVERSITY \\ UNIVERSITY OF OREGON \\ OSAKA UNIVERSITY
}

\author{
UNIVERSITY OF SOUTHERN CALIFORNIA \\ STANFORD UNIVERSITY \\ UNIVERSITY OF TOKYO \\ UNIVERSITY OF UTAH \\ WASHINGTON STATE UNIVERSITY \\ UNIVERSITY OF WASHINGTON

$* * *$
$*$
AMERICAN MATHEMATICAL SOCIETY
NAVAL WEAPONS CENTER

The Supporting Institutions listed above contribute to the cost of publication of this Journal, but they are not owners or publishers and have no responsibility for its content or policies.

Mathematical papers intended for publication in the Pacific Journal of Mathematics should be in typed form or offset-reproduced, (not dittoed), double spaced with large margins. Underline Greek letters in red, German in green, and script in blue. The first paragraph or two must be capable of being used separately as a synopsis of the entire paper. Items of the bibliography should not be cited there unless absolutely necessary, in which case they must be identified by author and Journal, rather than by item number. Manuscripts, in duplicate if possible, may be sent to any one of the four editors. Please classify according to the scheme of Math. Rev. Index to Vol. 39. All other communications to the editors should be addressed to the managing editor, or Elaine Barth, University of California, Los Angeles, California, 90024.

100 reprints are provided free for each article, only if page charges have been substantially paid. Additional copies may be obtained at cost in multiples of 50 .

The Pacific Journal of Mathematics is issued monthly as of January 1966. Regular subscription rate: $\$ 60.00$ a year (6 Vols., 12 issues). Special rate: $\$ 30.00$ a year to individual members of supporting institutions.

Subscriptions, orders for back numbers, and changes of address should be sent to Pacific Journal of Mathematics, 103 Highland Boulevard, Berkeley, California, 94708.

PUBLISHED BY PACIFIC JOURNAL OF MATHEMATICS, A NON-PROFIT CORPORATION

Printed at Kokusai Bunken Insatsusha (International Academic Printing Co., Ltd.), 270, 3-chome Totsuka-cho, Shinjuku-ku, Tokyo 160, Japan

* C. R. DePrima California Institute of Technology, Pasadena, CA 91109, will replace J. Dugundji until August 1974. 


\section{Pacific Journal of Mathematics}

Vol. 50, No. $2 \quad$ October, 1974

Mustafa Agah Akcoglu, John Philip Huneke and Hermann Rost, A counter example to the Blum Hanson theorem in general spaces .............

Huzihiro Araki, Some properties of modular conjugation operator of von

Neumann algebras and a non-commutative Radon-Nikodym theorem

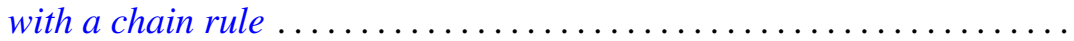

E. F. Beckenbach, Fook H. Eng and Richard Edward Tafel, Global properties of rational and logarithmico-rational minimal surfaces .....

David W. Boyd, A new class of infinite sphere packings ............. 383

K. G. Choo, Whitehead Groups of twisted free associative algebras ........

Charles Kam-Tai Chui and Milton N. Parnes, Limit sets of power series

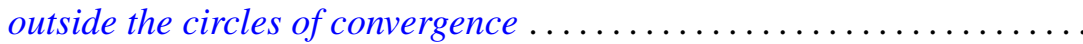

Allan Clark and John Harwood Ewing, The realization of polynomial

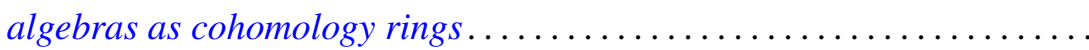

Dennis Garbanati, Classes of circulants over the p-adic and rational

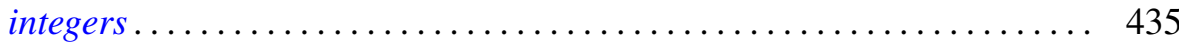

Arjun K. Gupta, On a "square" functional equation ................... 449

David James Hallenbeck and Thomas Harold MacGregor, Subordination and extreme-point theory ............................. 455

Douglas Harris, The local compactness of $v X \ldots \ldots . . . . . . . . . . . .4469$

William Emery Haver, Monotone mappings of a two-disk onto itself which fix the disk's boundary can be canonically approximated by homeomorphisms .................................. 477

Norman Peter Herzberg, On a problem of Hurwitz .................. 485

Chin-Shui Hsu, A class of Abelian groups closed under direct limits and subgroups formation ............................... 495

Bjarni Jónsson and Thomas Paul Whaley, Congruence relations and multiplicity types of algebras.....................

Lowell Duane Loveland, Vertically countable spheres and their wild sets.

Nimrod Megiddo, Kernels of compound games with simple components ....

Russell L. Merris, An identity for matrix functions ........ . .

E. O. Milton, Fourier transforms of odd and even tempered distributions ...

Dix Hayes Pettey, One-one-mappings onto locally connected generalized continua

Mark Bernard Ramras, Orders with finite global dimension

Doron Ravdin, Various types of local homogeneity. .

George Michael Reed, On metrizability of complete Moore spaces ...

Charles Small, Normal bases for quadratic extensions ..

Philip C. Tonne, Polynomials and Hausdorff matrices.... . . 